\title{
Definable Davies' theorem
}

by

\section{Asger Törnquist (Wien) and William Weiss (Toronto)}

\begin{abstract}
We prove the following descriptive set-theoretic analogue of a theorem of R. O. Davies: Every $\Sigma_{2}^{1}$ function $f: \mathbb{R} \times \mathbb{R} \rightarrow \mathbb{R}$ can be represented as a sum of rectangular $\Sigma_{2}^{1}$ functions if and only if all reals are constructible.
\end{abstract}

1. Introduction. In [1], R. O. Davies proved that the continuum hypothesis, $\mathrm{CH}$, is equivalent to the statement that every function $f: \mathbb{R} \times \mathbb{R} \rightarrow$ $\mathbb{R}$ can be represented as a sum of "rectangular" functions as follows: There are $g_{n}, h_{n}: \mathbb{R} \rightarrow \mathbb{R}, n \in \omega$, such that

$$
f(x, y)=\sum_{n=0}^{\infty} g_{n}(x) h_{n}(y)
$$

where at each $(x, y) \in \mathbb{R}^{2}$ there are at most finitely many non-zero terms in the above sum. We call such a representation a Davies representation of $f$. Thus Davies' Theorem says that $\mathrm{CH}$ is equivalent to the statement that every function $f: \mathbb{R} \times \mathbb{R} \rightarrow \mathbb{R}$ has Davies representation.

The purpose of this paper is to prove the following descriptive settheoretic analogue of Davies' Theorem:

TheOREM 1. Every $\Sigma_{2}^{1}$ function $f: \mathbb{R} \times \mathbb{R} \rightarrow \mathbb{R}$ has a Davies representation

$$
f(x, y)=\sum_{n=0}^{\infty} g(x, n) h(y, n),
$$

where $g, h: \mathbb{R} \times \omega \rightarrow \mathbb{R}$ are $\Sigma_{2}^{1}$ functions and the sum has only finitely many non-zero terms at each $(x, y) \in \mathbb{R}^{2}$, if and only if all reals are constructible.

We will also show that it is not possible to find a Davies representation of $f(x, y)=e^{x y}$ using Baire or Lebesgue measurable functions $g$ and $h$. Note though that $e^{x y}$ does have a representation as an infinite power series in $x$ 
and $y$. We will give an example of a Borel (in fact, $\Delta_{1}^{1}$ ) function $f: \mathbb{R} \times \mathbb{R} \rightarrow \mathbb{R}$ which does not admit a rectangular sum representation as above with Baire or Lebesgue measurable $g$ and $h$, even if we drop the pointwise finiteness condition on the sum, and only ask that at each $(x, y)$ the sum converges pointwise.

Organization. In $\S 2$ below we show (Theorem 2 ) that if there is a strongly $\Delta_{n}^{1}$ well-ordering of $\mathbb{R}$ then every $\Sigma_{n}^{1}$ function $f: \mathbb{R} \times \mathbb{R} \rightarrow \mathbb{R}$ admits a representation

$$
f(x, y)=\sum_{n=0}^{\infty} g(x, n) h(y, n),
$$

with $\Sigma_{n}^{1}$ functions $g, h: \mathbb{R} \times \omega \rightarrow \mathbb{R}$, and where the sum has only finitely many non-zero terms at each $(x, y) \in \mathbb{R}^{2}$.

In $\S 3$ we establish the converse to Theorem 2 in the case of $\Sigma_{2}^{1}$ functions (Theorem 3). We also establish a converse in the $\Sigma_{3}^{1}$ case, under the additional assumption that there is a measurable cardinal. Finally, we establish the two facts regarding representations using Baire and Lebesgue measurable functions mentioned after Theorem 1 above.

2. Inductive argument. The necessary descriptive set-theoretic background for this paper can be found in [9] and [8], in particular the definitions of the (lightface) point-classes $\Sigma_{n}^{1}, \Delta_{n}^{1}$ and $\Pi_{n}^{1}$. Here we recall the notions for $\Delta_{n}^{1}$ well-orderings that are the most important to us.

Following [2], we say that a $\Delta_{n}^{1}$ well-ordering $\prec$ of $\mathbb{R}$ is strongly $\Delta_{n}^{1}$ if it has length $\omega_{1}$ and the following (equivalent) statements hold (cf. [9, Chapter 5]):

1. If $P \subseteq \mathbb{R} \times \mathbb{R}$ is $\Sigma_{n}^{1}$ then

$$
R(x, y) \Leftrightarrow(\forall z \prec y) P(x, z)
$$

is $\Sigma_{n}^{1}$.

2. The initial segment relation IS $\subseteq \mathbb{R} \times \mathbb{R}^{\leq \omega}$ defined by

$$
\operatorname{IS}(x, y) \Leftrightarrow(\forall z \prec x)(\exists n) y(n)=z \wedge(\forall i, j) i=j \vee y(i) \neq y(j)
$$
is $\Sigma_{n}^{1}$.

If all reals are constructible then there is a strongly $\Delta_{2}^{1}$ well-ordering of $\mathbb{R}$ (see e.g. [6]).

It will often be necessary to work with recursively presented Polish spaces other than $\mathbb{R}$, such as $\omega^{\omega}$ or $\mathbb{R} \leq \omega$ (see below). Since all uncountable recursively presented Polish spaces are isomorphic in the sense that there is a $\Delta_{1}^{1}$ bijection between them with a $\Delta_{1}^{1}$ inverse (see $[9,3 \mathrm{E} .7]$ ), once we have a strongly $\Delta_{n}^{1}$ well-ordering of $\mathbb{R}$ we have a strongly $\Delta_{n}^{1}$ well-ordering of all recursively presented Polish spaces. For convenience we will use the same 
symbol, usually $\prec$, for such a well-ordering in all the recursively presented spaces we consider. This minor ambiguity poses no real danger.

We will say that a function $f: X \rightarrow Y$ from one recursively presented Polish space $X$ to another, $Y$, is $\Sigma_{n}^{1}$ (respectively $\Pi_{n}^{1}$ and $\Delta_{n}^{1}$ ) if its graph is $\Sigma_{n}^{1}$ (respectively $\Pi_{n}^{1}$ and $\Delta_{n}^{1}$ ). A function $f: \mathbb{R} \times \mathbb{R} \rightarrow \mathbb{R}$ is said to have a $\Sigma_{n}^{1}$ Davies representation if there are $\Sigma_{n}^{1}$ functions $g, h: \mathbb{R} \times \omega \rightarrow \mathbb{R}$ such that

$$
\sum_{n=0}^{\infty} g(x, n) h(y, n)
$$

and the sum has only finitely many non-zero terms at each $(x, y)$. The notions of $\Pi_{n}^{1}$ and $\Delta_{n}^{1}$ Davies representation are defined similarly.

THEOREM 2. If there is a strongly $\Delta_{n}^{1}$ well-ordering of $\mathbb{R}$ then every $\Sigma_{n}^{1}$ function $f: \mathbb{R} \times \mathbb{R} \rightarrow \mathbb{R}$ has a $\Sigma_{n}^{1}$ Davies representation. In particular, if all reals are constructible then every $\Sigma_{2}^{1}$ function has a $\Sigma_{2}^{1}$ Davies representation.

To prove this, we will need to verify that Davies' proof, which uses Zorn's Lemma, produces functions $g, h: \mathbb{R} \times \omega \rightarrow \mathbb{R}$ that are $\Sigma_{n}^{1}$ and witness that $f$ has a $\Sigma_{n}^{1}$ Davies representation. This in turn requires that we produce $\Sigma_{n}^{1}$ predicates (in the sense of [8, p. 3] or [6, p. 152-157]) that define $g$ and $h$. These predicates will essentially be formulas defining $g$ and $h$ by transfinite recursion as in the usual proof of the transfinite recursion theorem (see e.g. $[4$, p. $22,(2.6)])$.

If $X$ is a set, we write $X^{\leq \omega}$ for the set of functions $g: \alpha \rightarrow X$ for some $\alpha \in \omega+1$, and we set $\ln (g)=|\operatorname{dom}(g)|$, the cardinality of $\operatorname{dom}(g)$. For $g \in \mathbb{R} \leq \omega$ we let

$$
\operatorname{supp}(g)=\{n \in \omega: n \in \operatorname{dom}(g) \wedge g(n) \neq 0\} .
$$

It is convenient for the proof to work relative to a fixed countable sequence $x_{n} \in \mathscr{P}(\omega)$ of almost disjoint infinite subsets of $\omega$. The sequence $\left(x_{n}\right)$ will be used to make sure that certain almost disjoint families that are finite are not maximal, because they will be constructed so that they are almost disjoint from all $x_{n}, n \in \omega$. We will assume that the map $n \mapsto x_{n}$ is recursive.

Definition. The set $S \subseteq\left(\mathbb{R}^{\omega}\right)^{\leq \omega} \times\left(\mathbb{R}^{\omega}\right) \leq \omega$ is defined by $(g, h) \in S$ if and only if

(a) The sets $\operatorname{supp}(g(k)), \operatorname{supp}(h(m))$ and $x_{n}(k \in \operatorname{dom}(g), m \in \operatorname{dom}(h)$, $n \in \omega)$ form an almost disjoint sequence of sets.

(b) For all $m \in \operatorname{dom}(g)$ there are infinitely many $k$ such that $g(m)(k)=1$.

(c) For all $n \in \operatorname{dom}(h)$ there are infinitely many $k$ such that $h(n)(k)=1$. 
Note that $S$ is $\Delta_{1}^{1}$. We need the following lemma to encode the inductive step.

2.1. Lemma. Suppose $f \in \mathbb{R}^{\leq \omega}$ is given and $(g, h) \in S$ is such that $\operatorname{lh}(h)=\operatorname{lh}(f)$. Then there is $\theta=\theta(f, g, h): \omega \rightarrow \mathbb{R}$ such that:

(1) For all $k \in \operatorname{dom}(f)$,

$$
f(k)=\sum_{l=0}^{\infty} \theta(l) h(k)(l),
$$

and the sum has only finitely many non-zero terms.

(2) For all $n \in \operatorname{dom}(h), \operatorname{supp}(\theta) \cap \operatorname{supp}(h(n))$ is finite.

(3) For all $n \in \operatorname{dom}(g), \operatorname{supp}(\theta) \cap \operatorname{supp}(g(n))$ is finite.

(4) For all $n \in \omega, \operatorname{supp}(\theta) \cap x_{n}$ is finite.

(5) For infinitely many $k$ we have $\theta(k)=1$.

Moreover, $\theta$ may be found recursively in the given data. In particular, there is a $\Delta_{1}^{1}$ function $\theta: \mathbb{R}^{\leq \omega} \times S \rightarrow \mathbb{R}^{\omega}$ such that $\theta(f, g, h)$ satisfies (1)-(5) for all $(f, g, h) \in \mathbb{R}^{\leq \omega} \times S$.

Proof. We define by induction on $k \in \omega$ an increasing sequence $n_{k} \in \omega$ and $\theta\left\lceil n_{k}+1\right.$ such that

$\left(1^{\prime}\right)$ For all $m \in \operatorname{dom}(f) \cap(k+1)$,

$$
f(m)=\sum_{l=0}^{n_{m}} \theta(l) h(m)(l) .
$$

$\left(2^{\prime}\right)$ For all $m \in \operatorname{dom}(h) \cap(k+1), \operatorname{supp}\left(\theta\left\lceil n_{k}+1\right) \cap \operatorname{supp}(h(m)) \subseteq n_{m}+1\right.$.

$\left(3^{\prime}\right)$ For all $m \in \operatorname{dom}(g) \cap(k+1), \operatorname{supp}\left(\theta\left\lceil n_{k}+1\right) \cap \operatorname{supp}(g(m)) \subseteq n_{m}+1\right.$.

$\left(4^{\prime}\right)$ For all $m \leq k, \operatorname{supp}\left(\theta\left\lceil n_{k}+1\right) \cap x_{m} \subseteq n_{m}+1\right.$.

(5') $\theta\left(n_{k}\right)=1$.

Assuming this can be done, $\theta$ will be defined on all of $\omega$, since $n_{k}$ is increasing. By $\left(1^{\prime}\right)$ and $\left(2^{\prime}\right)$ it follows that for $m \in \operatorname{dom}(f)$ we will have

$$
f(m)=\sum_{l=0}^{\infty} \theta(l) h(m)(l)
$$

and by $\left(2^{\prime}\right)$ it is the case that $\theta(l) h(m)(l)=0$ for $l>n_{m}$. Thus (1) and (2) of the statement of the lemma hold. Finally, $\left(3^{\prime}\right),\left(4^{\prime}\right)$ and $\left(5^{\prime}\right)$ ensure (3), (4) and (5).

To see that we can satisfy $\left(1^{\prime}\right)-\left(5^{\prime}\right)$, suppose $n_{k}$ and $\theta\left\lceil n_{k}+1\right.$ have been defined.

CASE 1: $k+1 \notin \operatorname{dom}(f)$. Then we let $p>n_{k}$ be the least number greater than $n_{k}$ such that $p \notin \operatorname{supp}(g(m)), p \notin \operatorname{supp}(h(m))$ and $p \notin x_{m}$ for $m \leq k$. The number $p$ exists because of condition (a) in the definition of $S$. 
Define $n_{k+1}=p$, for $n_{k}<l<n_{k+1}$ let $\theta(l)=0$, and $\theta\left(n_{k+1}\right)=1$. Clearly conditions $\left(1^{\prime}\right)-\left(5^{\prime}\right)$ are satisfied.

CASE $2: k+1 \in \operatorname{dom}(f)$. Then let $p>n_{k}$ be the least number greater than $n_{k}$ such that $p \notin \operatorname{supp}(g(m)), p \notin \operatorname{supp}(h(m))$ and $p \notin x_{m}$ for $m \leq k$, and $h(k+1)(p)=1$. The number $p$ exists because of conditions (a) and (c) in the definition of $S$. We let $q>p$ be least such that $q \notin \operatorname{supp}(g(m))$, $q \notin \operatorname{supp}(h(m))$ and $q \notin x_{m}$ for $m \leq k+1$. Let $n_{k+1}=q$ and define, for $n_{k}<l \leq n_{k+1}$,

$$
\theta(l)= \begin{cases}1 & \text { if } l=q=n_{k+1}, \\ f(k+1)-\sum_{m=0}^{p-1} \theta(m) h(k+1)(m) & \text { if } l=p, \\ 0 & \text { otherwise. }\end{cases}
$$

It is easy to see that $\left(2^{\prime}\right)-\left(5^{\prime}\right)$ are satisfied. To see $\left(1^{\prime}\right)$, note that

$$
\begin{aligned}
\sum_{l=0}^{n_{k+1}} \theta(l) h(k+1)(l) & =\sum_{l=0}^{p} \theta(l) h(k+1)(l) \\
& =f(k+1)-\sum_{m=0}^{p-1} \theta(m) h(k+1)(m)+\sum_{l=0}^{p-1} \theta(l) h(k+1)(l) \\
& =f(k+1) .
\end{aligned}
$$

This ends Case 2. It is clear from the construction that $\theta$ is recursive in the given data $(f, g, h)$. Thus the map $(f, g, h) \mapsto \theta(f, g, h)$ is in particular $\Delta_{1}^{1}$.

2.2. Davies' argument as an inductive construction. For the remainder of this section of the paper, $\theta$ will be the function defined in Lemma 2.1. Using this lemma one can now produce a Davies representation of $f: \mathbb{R} \times \mathbb{R} \rightarrow \mathbb{R}$ by induction as follows: Assuming $\mathrm{CH}$, fix a well-ordering $\prec$ of $\mathbb{R}$ of order type $\omega_{1}$. Suppose $g, h:\{y \in \mathbb{R}: y \prec x\} \times \omega \rightarrow \mathbb{R}$ have been defined such that for all $y, z \prec x$,

$$
f(y, z)=\sum_{n=0}^{\infty} g(y, n) h(z, n)
$$

and that further if $\left(w_{m}\right)$ is an enumeration of the initial segment $\{y: y \prec x\}$ then the functions

$$
g_{0}(m)(n)=g\left(w_{m}, n\right) \quad \text { and } \quad h_{0}(m)(n)=h\left(w_{m}, n\right)
$$

are such that $\left(g_{0}, h_{0}\right) \in S$. If we let $f_{0}(m)=f\left(w_{m}, x\right)$ and define $g(x, n)=$ $\theta\left(f_{0}, g_{0}, h_{0}\right)(n)$ then it is easy to check using Lemma 2.1 that for $y \prec x$,

$$
f(x, y)=\sum_{n=0}^{\infty} g(x, n) h(y, n) .
$$


If $\left(w_{m}^{\prime}\right)$ enumerates $\{y: y \preceq x\}$ and we let $f_{1}(m)=f\left(x, w_{m}^{\prime}\right)$ and

$$
g_{1}(n)= \begin{cases}\theta\left(f_{0}, g_{0}, h_{0}\right) & \text { if } w_{n}^{\prime}=x, \\ g_{0}(k) & \text { if } w_{n}^{\prime}=w_{k},\end{cases}
$$

then $\left(h_{0}, g_{1}\right) \in S$, and if we let $\left.h(x, n)=\theta\left(f_{1}, h_{0}, g_{1}\right)\right)(n)$, it is again easy to check using the previous lemma that for all $y \preceq x$,

$$
f(y, x)=\sum_{n=0}^{\infty} g(y, n) h(x, n)
$$

Finally,

$$
g_{1}(m)(n)=g\left(w_{m}^{\prime}, n\right) \quad \text { and } \quad h_{1}(m)(n)=h\left(w_{m}^{\prime}, n\right)
$$

satisfy $\left(g_{1}, h_{1}\right) \in S$, thus allowing the induction to continue.

Our task is now to verify that if $\prec$ is a strongly $\Delta_{n}^{1}$ well-ordering of $\mathbb{R}$, then the construction we have described may be carried out in such a way that if $f: \mathbb{R} \times \mathbb{R} \rightarrow \mathbb{R}$ is $\Sigma_{n}^{1}$, then the functions $g, h: \mathbb{R} \times \omega \rightarrow \mathbb{R}$ will be $\Sigma_{n}^{1}$. This can be done since the strongly $\Delta_{n}^{1}$ well-ordering allows us to enumerate initial segments in a uniformly $\Delta_{n}^{1}$ way. However, in order to be able to write down $\Sigma_{n}^{1}$ definitions of $g$ and $h$ we need a lemma which says that there is a $\Sigma_{n}^{1}$ function which can correctly compute $g\lceil\{y: y \prec x\} \times \omega$ and $h\lceil\{y: y \prec x\} \times \omega$ for every $x$.

Before stating that lemma we introduce various functions and predicates. Fix a strongly $\Delta_{n}^{1}$ well-ordering $\prec$ of $\mathbb{R}$ and let IS $\subseteq \mathbb{R} \times \mathbb{R} \leq \omega$ be the initial segment relation as defined at the beginning of this section. Define IS* : $\mathbb{R} \rightarrow \mathbb{R} \leq \omega$ by

$$
\operatorname{IS}^{*}(x)=y \Leftrightarrow \operatorname{IS}(x, y) \wedge(\forall z \prec y) \neg \operatorname{IS}(x, z) .
$$

Note that IS* is $\Delta_{n}^{1}$. We also define a partial function IS $^{\#}: \mathbb{R} \times \mathbb{R} \rightarrow \omega$ by

$$
\operatorname{IS}^{\#}(x, y)=n \Leftrightarrow \operatorname{IS}^{*}(x)(n)=y .
$$

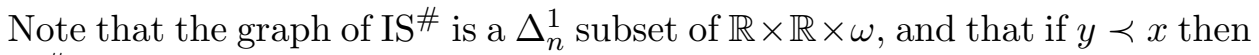
$\operatorname{IS}^{\#}(x, y)$ computes the unique $n$ which $y$ corresponds to in the enumeration of the initial segment of $x$ given by IS* $(x)$. Finally, we define

$$
\operatorname{succ}(x)=y \Leftrightarrow(\forall z \prec y) z=x \vee z \prec x .
$$

2.3. Lemma. Let $f: \mathbb{R} \times \mathbb{R} \rightarrow \mathbb{R}$ be $\Sigma_{n}^{1}$ and suppose there is a strongly $\Delta_{n}^{1}$ well-ordering $\prec$ of $\mathbb{R}$. Then there is a unique $\Sigma_{n}^{1}$ function $F: \mathbb{R} \rightarrow$ $\left(\mathbb{R}^{\omega}\right) \leq \omega \times\left(\mathbb{R}^{\omega}\right) \leq \omega$ satisfying $F(x)=(G, H)$ if and only if

(1) $\operatorname{lh}(G)=\operatorname{lh}(H)=\operatorname{lh}\left(\operatorname{IS}^{*}(x)\right)$ and $(G, H) \in S$.

(2) If $z, z^{\prime} \prec x$, $\operatorname{IS}^{\#}(x, z)=k$ and $\operatorname{IS}^{\#}\left(x, z^{\prime}\right)=k^{\prime}$ then

$$
f\left(z, z^{\prime}\right)=\sum_{n=0}^{\infty} G(k)(n) H\left(k^{\prime}\right)(n) .
$$


(3) For all $y \prec x$, if we let $w^{\prime}=\operatorname{IS}^{*}(y), w=\operatorname{IS}^{*}(x)$ and $f_{0}(k)=$ $f\left(y, w^{\prime}(k)\right)$, and define, for $k \in \operatorname{dom}\left(w^{\prime}\right)$,

$$
G^{\prime}(k)=G(l) \Leftrightarrow w^{\prime}(k)=w(l)
$$

and

$$
H^{\prime}(k)=H(l) \Leftrightarrow w^{\prime}(k)=w(l),
$$

then $w(m)=y$ implies that

$$
G(m)=\theta\left(f_{0}, G^{\prime}, H^{\prime}\right) .
$$

(4) For all $y \prec x$, if we let $w^{\prime}=\operatorname{IS}^{*}(y), w^{\prime \prime}=\operatorname{IS}^{*}(\operatorname{succ}(y)), w=\operatorname{IS}^{*}(x)$ and $f_{1}(k)=f\left(w^{\prime \prime}(k), y\right)$, and define, for $k \in \operatorname{dom}\left(w^{\prime \prime}\right)$,

$$
G^{\prime \prime}(k)=G(l) \Leftrightarrow w^{\prime \prime}(k)=w(l),
$$

and for $k \in \operatorname{dom}\left(w^{\prime}\right)$,

$$
H^{\prime}(k)=H(l) \Leftrightarrow w^{\prime}(k)=w(l),
$$

then $w(m)=y$ implies that

$$
H(m)=\theta\left(f_{1}, H^{\prime}, G^{\prime \prime}\right) .
$$

Proof. Conditions (1)-(4) express exactly that for $y \prec x$, if we let

$$
g(y, n)=G\left(\operatorname{IS}^{\#}(x, y)\right)(n) \quad \text { and } h(y, n)=H\left(\operatorname{IS}^{\#}(x, y)\right)(n)
$$

then $g$ and $h$ are the functions we have constructed at stage $x$ in the inductive construction described in 2.2 above, provided that at any stage of the induction we use the enumeration of the initial segments given by the function IS*. Thus $F$ is unique and defined for all $x$. Finally, we note that conditions (1)-(4) can be expressed using $\Sigma_{n}^{1}$ predicates when $f$ is a $\Sigma_{n}^{1}$ function. For instance, (3) may be replaced by

$$
\begin{aligned}
& (\forall y \prec x)\left(\exists w, w^{\prime}, f_{0}, G^{\prime}, H^{\prime} \in \mathbb{R}^{\leq \omega}\right)\left(w^{\prime}=\operatorname{IS}^{*}(y) \wedge w=\mathrm{IS}^{*}(x)\right. \\
& \wedge \operatorname{lh}\left(f_{0}\right)=\operatorname{lh}\left(w^{\prime}\right) \wedge\left(\forall k \in \operatorname{dom}\left(w^{\prime}\right)\right)\left(f_{0}(k)=f\left(y, w^{\prime}(k)\right)\right. \\
& \left.\wedge(\forall l \in \operatorname{dom}(G))\left(G^{\prime}(k)=G(l) \wedge H^{\prime}(k)=H(l) \Leftrightarrow w^{\prime}(k)=w(l)\right)\right) \\
& \left.\wedge(\forall m \in \operatorname{dom}(w))\left(w(m) \neq y \vee G(m)=\theta\left(f_{0}, G^{\prime}, H^{\prime}\right)\right)\right) .
\end{aligned}
$$

Thus (1)-(4) gives a $\Sigma_{n}^{1}$ definition of the graph of $F$, and so the function $F$ is $\Sigma_{n}^{1}$.

Proof of Theorem 2. If $f: \mathbb{R} \times \mathbb{R} \rightarrow \mathbb{R}$ is $\Sigma_{n}^{1}$ and $\prec$ is a strongly $\Delta_{n}^{1}$ well-ordering, let $F$ be as in Lemma 2.3 and let $F(x)=(G(x), H(x))$ for all $x$. Then

$$
g(x, n)=G(\operatorname{succ}(x))\left(\operatorname{IS}^{\#}(\operatorname{succ}(x), x)\right)(n)
$$

and

$$
h(x, n)=H(\operatorname{succ}(x))\left(\operatorname{IS}^{\#}(\operatorname{succ}(x), x)\right)(n)
$$

define $\Sigma_{n}^{1}$ functions that give us a Davies representation of $f$. 
REMARK. If $f: \mathbb{R} \times \mathbb{R} \rightarrow \mathbb{R}$ is $\Delta_{n}^{1}$ then conditions (1)-(4) define a $\Delta_{n}^{1}$ function $F$. Consequently, the functions $g$ and $h$ produced in the proof of Theorem 2 will be $\Delta_{n}^{1}$. Therefore we have:

2.4. Corollary. If there is a strongly $\Delta_{n}^{1}$ well-ordering of $\mathbb{R}$ then every $\Delta_{n}^{1}$ function $f: \mathbb{R} \times \mathbb{R} \rightarrow \mathbb{R}$ has a $\Delta_{n}^{1}$ Davies representation.

3. A definable converse. We now show the following converse to Theorem 2 for $\Sigma_{2}^{1}$ functions:

TheOREM 3. If there are $\Sigma_{2}^{1}$ functions $g, h: \mathbb{R} \times \omega \rightarrow \mathbb{R}$ such that

$$
e^{x y}=\sum_{n=0}^{\infty} g(x, n) h(y, n)
$$

with only finitely many non-zero terms at each $(x, y)$ then there is a $\Sigma_{2}^{1}$ well-ordering of $\mathbb{R}$.

Since by Mansfield's Theorem ([7], see also [4, 25.39]) the existence of a $\Sigma_{2}^{1}$ well-ordering or $\mathbb{R}$ is equivalent to the statement that all reals are constructible, Theorem 3 together with Theorem 2 proves Theorem 1 as stated in the introduction. The proof requires several lemmata: Then

3.1. Lemma. Let $b_{0}, \ldots, b_{n} \in \mathbb{R}$ be distinct reals and $c_{0}, \ldots, c_{n} \in \mathbb{R}$.

$$
f(x)=\sum_{j=0}^{n} c_{j} e^{x b_{j}}
$$

has $n+1$ distinct roots if and only if $c_{0}=\cdots=c_{n}=0$.

Proof. By induction on $n$. If $f(x)$ has $n+1$ distinct roots then so does

$$
g(x)=e^{-b_{0} x} f(x) .
$$

Using Rolle's Theorem from calculus it follows that $g^{\prime}(x)$ has $n$ distinct roots, and so by the inductive hypothesis must be constantly zero. Thus $f(x)$ is constantly zero.

3.2. LemMA. Let $a_{0}, \ldots, a_{n}$ and $b_{0}, \ldots, b_{n}$ be two distinct sequences of real numbers. Then there are no functions $g_{l}, h_{l}: \mathbb{R} \rightarrow \mathbb{R}, l<n$, such that

$$
e^{a_{i} b_{j}}=\sum_{l=0}^{n-1} g_{l}\left(a_{i}\right) h_{l}\left(b_{j}\right)
$$

for all $0 \leq i, j \leq n$. 
Proof. If there are such functions then we have the matrix identity

$$
\left[e^{a_{i} b_{j}}\right]=\left[\begin{array}{ccc}
g_{0}\left(a_{0}\right) & \cdots & g_{n-1}\left(a_{0}\right) \\
\vdots & & \vdots \\
g_{0}\left(a_{n}\right) & \cdots & g_{n-1}\left(a_{n}\right)
\end{array}\right]\left[\begin{array}{ccc}
h_{0}\left(b_{0}\right) & \cdots & h_{0}\left(b_{n}\right) \\
\vdots & & \vdots \\
h_{n-1}\left(b_{0}\right) & \cdots & h_{n-1}\left(b_{n}\right)
\end{array}\right]
$$

and so $\left[e^{a_{i} b_{j}}\right]$ is a product of an $(n+1) \times n$ and an $n \times(n+1)$ matrix. It follows that $\operatorname{rank}\left(\left[e^{a_{i} b_{j}}\right]\right) \leq n$, which contradicts the previous lemma.

3.3. Lemma. Assume $\Sigma_{n}^{1}$ uniformization holds and that there are $\Sigma_{n}^{1}$ functions $g, h: \mathbb{R} \times \omega \rightarrow \mathbb{R}$ such that

$$
e^{x y}=\sum_{n=0}^{\infty} g(x, n) h(y, n)
$$

with only finitely many non-zero terms at each $(x, y)$. Suppose there is an uncountable $\Sigma_{n}^{1}$ set $A \subseteq \mathbb{R}$ and a binary $\Sigma_{n}^{1}$ relation $\prec$ on $\mathbb{R}$ such that $(A, \prec)$ is well-ordered. Then there is a $\Sigma_{n}^{1}$ well-ordering of $\mathbb{R}$.

Proof. Define

$$
N(x, y)=k \Leftrightarrow g(x, k) h(y, k) \neq 0 \wedge(\forall l>k) g(x, l) h(y, l)=0 .
$$

Clearly $N: \mathbb{R} \times \mathbb{R} \rightarrow \omega$ is $\Sigma_{n}^{1}$. Also define $Q \subseteq \mathbb{R} \times \omega$ by

$$
\begin{aligned}
Q(x, n) \Leftrightarrow & \left(\exists a \in \mathbb{R}^{\omega}\right)(\forall i)(\forall j)(i=j \vee a(i) \neq a(j)) \\
& \wedge(\forall k)(a(k) \in A \wedge N(x, a(k))=n),
\end{aligned}
$$

which is $\Sigma_{n}^{1}$. Let $Q^{*}: \mathbb{R} \rightarrow \omega$ be a $\Sigma_{n}^{1}$ uniformization of $Q$. Note that $Q^{*}$ is defined everywhere since $A$ is uncountable.

Now define $R \subseteq \mathbb{R} \times[\mathbb{R}]^{<\omega}$, where $[\mathbb{R}]^{<\omega}$ denotes the set of finite subsets $\left({ }^{1}\right)$ of $\mathbb{R}$, by

$$
R(x, s) \Leftrightarrow|s|=Q^{*}(x)+2 \wedge(\forall y \in s)\left(y \in A \wedge N(x, y)=Q^{*}(x)\right) .
$$

Let $R^{*}: \mathbb{R} \rightarrow[\mathbb{R}]^{<\omega}$ be a $\Sigma_{n}^{1}$ uniformization of $R$.

Claim. $R^{*}$ is finite-to-one.

Proof. Suppose not. Then there is some $s=\left\{b_{0}, \ldots, b_{n}\right\}$ such that $R^{*-1}(s)$ is infinite. Pick $a_{0}, \ldots, a_{n} \in R^{*-1}(s)$ distinct. Note that since $R^{*}\left(b_{i}\right)=s$ we have $Q^{*}\left(b_{i}\right)=|s|-2=n-1$. Thus

$$
e^{a_{i} b_{j}}=\sum_{l=0}^{n-1} g\left(a_{i}, l\right) h\left(b_{j}, l\right),
$$

contradicting the previous lemma.

$\left({ }^{1}\right)$ Formally we let $[\mathbb{R}]^{<\omega}=\left\{s \in \mathbb{R}^{<\omega}:(\forall k<\operatorname{lh}(s)-1) s(k)<s(k+1)\right\}$, where $<$ is the usual linear ordering of $\mathbb{R}$. Note that for $s \in[\mathbb{R}]^{<\omega}$, the quantifiers $(\forall x \in s)$ and $(\exists x \in s)$ can be replaced by number quantifiers in hierarchy calculations. 
Let $\prec_{\text {lex }}$ be the lexicographic order on $[A]^{<\omega}$ that we obtain from the well-ordering $\prec$ of $A$. Then we define $<^{*}$ by

$$
x<^{*} y \Leftrightarrow R^{*}(x) \prec_{\text {lex }} R^{*}(y) \vee\left(R^{*}(x)=R^{*}(y) \wedge x<y\right),
$$

where $<$ is the usual linear ordering of $\mathbb{R}$. Since $R^{*}$ is finite-to-one, $<^{*}$ is a $\Sigma_{n}^{1}$ well-ordering of $\mathbb{R}$.

3.4. Lemma. There are no Baire or Lebesgue measurable functions $g, h: \mathbb{R} \times \omega \rightarrow \mathbb{R}$ such that

$$
e^{x y}=\sum_{n=0}^{\infty} g(x, n) h(y, n)
$$

where the sum has finitely many non-zero terms at each $(x, y)$.

Proof. Suppose there are Baire measurable $g, h: \mathbb{R} \times \omega \rightarrow \mathbb{R}$ representing $e^{x y}$ as above. Then $N: \mathbb{R} \times \mathbb{R} \rightarrow \omega$ defined by

$$
N(x, y)=k \Leftrightarrow g(x, k) h(y, k) \neq 0 \wedge(\forall l>k) g(x, l) h(y, l)=0
$$

is also Baire measurable. It follows that there is some $n_{0}$ such that

$$
A=\left\{(x, y) \in \mathbb{R}^{2}: N(x, y)=n_{0}\right\}
$$

is non-meagre and has the property of Baire. Thus we may find $U, V \subseteq \mathbb{R}$ open and non-empty such that $A$ is comeagre in $U \times V$. By KuratowskiUlam's Theorem it follows that

$$
\left\{x \in U: A_{x} \text { is comeagre in } V\right\}
$$

is comeagre in $U$. Hence we may pick distinct elements $a_{0}, \ldots, a_{n_{0}+1} \in U$ such that the section $A_{a_{i}}$ is comeagre in $V$ for all $i=0, \ldots, n_{0}+1$. But then we can find distinct elements

$$
b_{0}, \ldots, b_{n_{0}+1} \in \bigcap_{i=0}^{n_{0}+1} A_{a_{i}},
$$

which gives us that for $0 \leq i, j \leq n_{0}+1$,

$$
e^{a_{i} b_{j}}=\sum_{n=0}^{n_{0}} g\left(a_{i}, n\right) h\left(b_{j}, n\right),
$$

contradicting Lemma 3.2.

The proof of the Lebesgue measurable case is similar.

Proof of Theorem 3. Suppose we have $\Sigma_{2}^{1}$ functions $g, h: \mathbb{R} \times \omega \rightarrow \mathbb{R}$ representing $e^{x y}$. By the previous lemma, $g$ and $h$ cannot be Baire measurable, and so $L \cap \mathbb{R}$ cannot be countable by [4, 26.21]. But then we can apply Lemma 3.3 with $A=L \cap \mathbb{R}$ and $\prec$ the canonical $\Sigma_{2}^{1}$ well-ordering of $L \cap \mathbb{R}$ to get a $\Sigma_{2}^{1}$ well-ordering of $\mathbb{R}$. 
Remark. Assume $\Sigma_{3}^{1}$ uniformization. Suppose there is a measurable cardinal and let $U$ be a normal ultrafilter witnessing this. Then the tree representation for $\Sigma_{3}^{1}$ (see [6, p. 201], also [4, 32.14]) and [6, 15.10] imply that if $\mathbb{R} \cap L[U]$ is countable then all $\Sigma_{3}^{1}$ functions have the property of Baire. Since by $[11,4.6]$ there is a $\Sigma_{3}^{1}$ well-ordering of $\mathbb{R} \cap L[U]$, the proof above then shows that if there is a $\Sigma_{3}^{1}$ Davies representation of $e^{x y}$ then there is a $\Sigma_{3}^{1}$ well-ordering of $\mathbb{R}$. In fact, we obtain the following stronger result:

3.5. Corollary. Assume $\Sigma_{3}^{1}$ uniformization. Suppose there is a measurable cardinal and let $U$ be a normal ultrafilter witnessing this. Then if there are $\Sigma_{3}^{1}$ functions $g, h: \mathbb{R} \times \omega \rightarrow \mathbb{R}$ such that

$$
e^{x y}=\sum_{n=0}^{\infty} g(x, n) h(y, n)
$$

with only finitely many non-zero terms at each $(x, y)$ then $\mathbb{R}=\mathbb{R} \cap L[U]$ and so there is a strongly $\Delta_{3}^{1}$ well-ordering of $\mathbb{R}$.

Proof. By inspecting the proof of Lemma 3.3, there exists a finite-to-one $\Sigma_{3}^{1}$ function $\theta: \mathbb{R} \rightarrow \mathbb{R} \cap L[U]$. Since the relation $R \subseteq \mathbb{R} \times \mathbb{N}$ defined by

$$
R(y, n) \Leftrightarrow\left(\exists x_{1}, \ldots, x_{n}\right) \bigwedge_{i=1}^{n} \theta\left(x_{i}\right)=y \wedge \bigwedge_{i \neq j} x_{i} \neq x_{j}
$$

is $\Sigma_{3}^{1}$, it is absolute for transitive models containing $U$. Suppose that there is $x_{1} \in \mathbb{R} \backslash L[U]$ and let $y=\theta\left(x_{1}\right)$. If $n=\left|\theta^{-1}(y) \cap L[U]\right|$ then $R(y, n+1)$ holds in $V$. By absoluteness it holds in $L[U]$, contradicting the fact that $n=\left|\theta^{-1}(y) \cap L[U]\right|$. Thus $\mathbb{R}=\mathbb{R} \cap L[U]$ and by $[11,5.2]$ there is a $\Delta_{3}^{1}$ well-ordering of $\mathbb{R}$.

In light of Theorem 3, it is natural to ask the following:

Question 1. If there are $\Sigma_{2}^{1}$ functions $g_{n}, h_{n}, n \in \omega$, such that

$$
e^{x y}=\sum_{n=0}^{\infty} g_{n}(x) h_{n}(y)
$$

with the sum having only finitely many non-zero terms at each $(x, y)$, does the conclusion of Theorem 3 still hold? That is, is it necessary in Theorem 3 that $g_{n}, h_{n}$ are $\Sigma_{2}^{1}$ uniformly in $n$ ?

In [10], Shelah shows that the converse in Davies' original theorem does not remain true if we drop the assumption that the sum must have at most finitely many non-zero terms and only require the sum to converge pointwise. In a similar vein we ask: 
Question 2. If we drop the finiteness condition, does Theorem 1 still hold?

Shelah also shows in [10] that if we add $\aleph_{2}$ Cohen reals then there is a function $f: \mathbb{R} \times \mathbb{R} \rightarrow \mathbb{R}$ which does not allow a representation

$$
f(x, y)=\sum_{n}^{\infty} g_{n}(x) h_{n}(y)
$$

even when we allow for the sum to have infinitely many non-zero terms, requiring only that it converges pointwise. As a counterpoint to that result, we point out the following:

TheOREM 4. There is a Borel function $f: \mathbb{R} \times \mathbb{R} \rightarrow \mathbb{R}$ such that for no $g_{n}, h_{n}: \mathbb{R} \rightarrow \mathbb{R}$ that are Baire measurable do we have

$$
f(x, y)=\sum_{n=0}^{\infty} g_{n}(x) h_{n}(y)
$$

for all $(x, y) \in \mathbb{R}^{2}$, where the sum converges pointwise, but may have infinitely many non-zero terms. The same holds if we replace Baire measurable by Lebesgue measurable.

Proof. Let as usual $E_{0}$ denote the equivalence relation on $2^{\omega}$ defined by

$$
x E_{0} y \Leftrightarrow(\exists N)(\forall n \geq N) x(n)=y(n) .
$$

Let $\mathbf{1}_{E_{0}}$ be the characteristic function of $E_{0}$. Suppose now that there are Baire measurable $g_{n}, h_{n}: \mathbb{R} \rightarrow \mathbb{R}$ such that

$$
\mathbf{1}_{E_{0}}(x, y)=\sum_{n=0}^{\infty} g_{n}(x) h_{n}(y) .
$$

Then we can find a dense $G_{\delta}$ set $A$ on which all the functions $g_{n}$ and $h_{n}$ are continuous. But then for $x, y \in A$ we have

$$
x E_{0} y \Leftrightarrow(\forall k)(\exists N \geq k) \sum_{n=0}^{N} g_{n}(x) h_{n}(y)>\frac{1}{2} .
$$

This gives us a $G_{\delta}$ definition of $E_{0}$ on $A$, and hence $E_{0}$ must be a smooth equivalence relation on $A$ by [3, Corollary 1.2]. But $E_{0}$ is not smooth on any comeagre set, and we have a contradiction.

The proof of the Lebesgue measurable case is similar.

Remark. By [5] (see also [4, Exercise 26.2]), if there is a Cohen real (respectively random real) over $L$ in $V$, then all $\Delta_{2}^{1}$ functions are Baire measurable (respectively Lebesgue measurable). Thus it follows that in this setting, $\mathbf{1}_{E_{0}}$ cannot be represented as an infinite pointwise convergent sum of rectangular $\Delta_{2}^{1}$ functions. 
Acknowledgements. A. Törnquist was supported in part by the Danish Natural Sciences Research Council post-doctoral grant no. 272-06-0211.

\section{References}

[1] R. O. Davies, Representation of functions of two variables as sums of rectangular functions I, Fund. Math. 85 (1974), 177-183.

[2] L. Harrington, $\Pi_{2}^{1}$ sets and $\Pi_{2}^{1}$ singletons, Proc. Amer. Math. Soc. 52 (1975), 356360 .

[3] L. Harrington, A. Kechris and A. Louveau, A Glimm-Effros dichotomy for Borel equivalence relations, J. Amer. Math. Soc. 3 (1990), 903-928.

[4] T. Jech, Set Theory, Springer, Berlin, 2003.

[5] H. Judah and S. Shelah, $\Delta_{2}^{1}$-sets of reals, Ann. Pure Appl. Logic 42 (1989), 207-223.

[6] A. Kanamori, The Higher Infinite, Springer, Berlin, 1997.

[7] R. Mansfield, The non-existence of $\Sigma_{2}^{1}$ well-orderings of the Cantor set, Fund. Math. 86 (1985), 279-282.

[8] R. Mansfield and G. Weitkamp, Recursive Aspects of Descriptive Set Theory, Oxford Univ. Press, New York, 1985.

[9] Y. N. Moschovakis, Descriptive Set Theory, North-Holland, 1980.

[10] S. Shelah, On Ciesielski's problems, J. Appl. Anal. 3 (1997), 191-209.

[11] J. H. Silver, Measurable cardinals and $\Delta_{3}^{1}$ well-orderings, Ann. of Math. (2) 94 (1971), 414-446.

Kurt Gödel Research Center

University of Vienna

Währinger Strasse 25

1090 Wien, Austria

E-mail: asger@logic.univie.ac.at
Department of Mathematics University of Toronto 40 St. George Street, Room 6092 Toronto, Ontario, Canada E-mail: weiss@math.utoronto.ca

Received 23 February 2008;

in revised form 15 June 2009 